\title{
Der Paradigmenmacher. Nachruf auf Immanuel Wallerstein (1930-2019)
}

\author{
Stefan Schmalz
}

Online publiziert: 27. April 2020

(C) Der/die Autor(en) 2020

Immanuel Wallerstein starb am 31. August 2019 im Alter von 88 Jahren. Sein mehrbändiges Werk zum modernen Weltsystem gilt als Klassiker der Soziologie. Er war Teil einer Gruppe von Soziologen, die sich im Umfeld der 1968er-Bewegung kritisch mit Strukturen, Abhängigkeiten und sozialen Bewegungen im globalen Kapitalismus auseinandersetzten und so zu Weltruhm gelangten. Oft wurde von einer ,gang of four" gesprochen, der neben Wallerstein seine Kollegen Samir Amin, Giovanni Arrighi und Andre Gunder Frank angehörten (Amin et al. 1982, 1990). Dabei war sich die Gang keineswegs immer einig; Wallersteins Thesen waren ebenso streitbar wie er selbst. Doch selbst für jene, die nicht alle seiner Auffassungen teilen oder - wie ich selbst - eher einer anderen Perspektive der Weltsystemanalyse anhängen, sind seine Thesen unbestreitbar eine wichtige, ja unverzichtbare Referenz. Und das gilt für eine unüberschaubare Fülle von Studien, die globale Zusammenhänge und Abhängigkeiten behandeln.

Immanuel Maurice Wallerstein wurde am 28. September 1930 in New York geboren. Seine polnisch-jüdischen Eltern waren im Jahr 1923 von Berlin in die USA ausgewandert, sein Vater war Arzt. Seine Familie beschrieb Wallerstein (2000, S. XV) später als ,,politisch bewusst“" und antifaschistisch. Wallerstein entschied sich für ein Studium an der Columbia University in New York. In seiner Studienzeit war er im World Assembly of Youth (WAY) aktiv und setzte sich in dieser Zeit auch kritisch mit dem Realsozialismus auseinander, war aber gleichzeitig ein entschiedener Gegner des McCarthyism, über den er seine Master-Arbeit verfasste. Sein Kontakt mit den afrikanischen Delegierten des WAY entfachte sein Interesse an den afrikanischen Unabhängigkeitsbewegungen. Sein wissenschaftlicher Fokus lag darum auch für rund zwei Jahrzehnte im Bereich der Afrika-Forschung: Im Jahr

S. Schmalz ( $\square)$

Friedrich-Schiller-Universität Jena, Jena, Deutschland

E-Mail: s.schmalz@uni-jena.de 
1959 promovierte er mit einer vergleichenden Arbeit zur Nationalstaatswerdung in Ghana und der Elfenbeinküste (Wallerstein 1964), von 1973 bis 1975 war er sogar Präsident der African Studies Association. Im Zuge seiner Auseinandersetzung mit den afrikanischen Unabhängigkeitsbewegungen wuchs jedoch seine Skepsis gegenüber modernisierungstheoretischen und nationalstaatszentrierten, zugleich aber auch gegenüber orthodox-marxistischen Argumentationsmustern. So kam er auf seine holistische Methode, die er als Weltsystemanalyse bezeichnete.

Die Wurzeln seines Ansatzes lassen sich zu vier Diskussionssträngen zurückverfolgen (Wallerstein 2007, S. 11): der lateinamerikanischen Dependenztheorie und der Diskussion um räumliche Ungleichheit im globalen Kapitalismus (Zentrum-Peripherie-Modell); der Debatte um den Marx'schen Begriff der asiatischen Produktionsweise und der Kritik an historischen Stufenmodellen gesellschaftlicher Entwicklung; den Studien des französischen Historikers Fernand Braudel zu den Strukturen der „longue durée“ in der Geschichte; sowie der Dobb-Sweezy-Debatte über die Ursprünge des Kapitalismus, aus der er Sweezys Position übernahm, dass der Fernhandel als Treibhaus kapitalistischer Entwicklung diente. Das Ergebnis seiner Überlegungen bildete sein vierbändiges Hauptwerk The Modern World System, dessen erster Band Capitalist Agriculture and the Origins of the European World-Economy in the Sixteenth Century im Jahr 1974 erschien (Wallerstein 2004). Eine wichtige Innovation dieser Arbeiten lag darin, mit dem Weltsystem einen globalen Analyserahmen für gesellschaftliche Prozesse zu entwickeln und somit bereits früh den „methodologischen Nationalismus“ der Sozialwissenschaften herauszufordern. Denn Wallerstein definiert das moderne Weltsystem als eine kapitalistisch organisierte Weltwirtschaft, die eine dezentralisierte politische Struktur aufweist und sich im Gegensatz zu früheren Systemen über den gesamten Globus erstreckt (Wallerstein 1979, S. 19f.). Wallerstein wurde außerdem vom Strukturfunktionalismus eines Talcott Parsons beeinflusst (Parsons 1972), auch wenn er selbst das abstritt. Gleichwohl beschreibt Wallerstein wie Parsons Systeme, die einer spezifischen Logik, z.B. säkularen Trends, folgen, und weist einzelnen Elementen in diesen Systemen, darunter Staaten, Funktionen zu.

Das moderne Weltsystem entstand Wallerstein zufolge im ,langen 16. Jahrhundert" mit der Kolonisation Lateinamerikas. Es tendiert seitdem zur Expansion und ist durch eine räumliche Hierarchie geprägt. Die Inkorporation der „Neuen Welt“ trug zu einem stetigen Ressourcentransfer nach Nordwesteuropa und zur Herausbildung von Zentrum-Peripherie-Verhältnissen bei. Zur Ausdifferenzierung dieser Strukturen führte Wallerstein das Konzept der Semiperipherie ein, die als ein Brückenkopf zwischen Zentrum und Peripherie eine Art geografische Mittelschicht im höchst ungleichen Weltsystem darstellt (Wallerstein 2007, S. 23 ff.). Wallerstein (2000, S. 253 ff.) analysierte zudem historische Hegemoniezyklen im Weltsystem, die jeweils von einer Ordnungsmacht strukturiert werden (Habsburger, Niederlande, Großbritannien und USA) und die weltpolitische Dynamik prägen. Diese Überlegungen bilden heute einen wesentlichen Bezugspunkt der Debatte um den Aufstieg Chinas (Schmalz 2018, S. 27 ff.). In seinem späteren Werk widmete sich Wallerstein weiteren Analyseeinheiten und Dynamiken im modernen Weltsystem. Er beschrieb etwa, dass mit der Durchsetzung des Liberalismus gegen Ende des 18. Jahrhunderts eine Geokultur entstanden sei, deren Ideen, Normen und Werte beinahe weltweit geteilt werden 
(Wallerstein 2011). Er arbeitete zudem heraus, dass Haushalte als die zentralen Wirtschaftseinheiten der Weltwirtschaft zu betrachten seien. Diese verfügen neben der Lohnarbeit oft über andere Einkommensformen, etwa aus der Subsistenzproduktion, die je nach Zone im Weltsystem variieren (Wallerstein 2007, S. 32 ff.; Schmalz et al. 2017). Im Dialog mit Étienne Balibar hob Wallerstein ferner hervor, wie das Zusammenspiel von Klasse, Nation und Rasse globale Ungleichheitsverhältnisse strukturiert (Balibar und Wallerstein 1991) - ihr Begriff des kulturellen Rassismus spielt heute in der Debatte um rechtspopulistische Kräfte in Europa eine wichtige Rolle.

Wallersteins Versuch, diese Überlegungen in einem einheitlichen Theoriegebäude zusammenzuführen, mündete in die eindrucksvolle Systemarchitektur, die uns heute vorliegt. Gleichzeitig trug Wallersteins Bemühen um Geschlossenheit mitunter dazu bei, dass einzelne Konzepte (antisystemische Bewegungen, starke und schwache Staaten etc.) etwas holzschnittartig anmuten. Eine Folge war, dass einige seiner intellektuellen Weggefährten eigene Wege gingen: Andre Gunder Frank (1998, S. 266) bezweifelte etwa die historischen Grundlagen des Wallerstein'schen Weltsystems und konstruierte einen 5000-Jahres-Zeitraum mit ökonomischen Zyklen, in denen der moderne Kapitalismus lediglich eine Nebenrolle spielt. Giovanni Arrighi (1994) tat genau das Gegenteil: Er versuchte, den Begriff des Weltsystems zu vermeiden, und wandte sich aus einer historisch-vergleichenden Perspektive der Anatomie der einzelnen historischen Welthegemonien $\mathrm{zu}$.

Wallerstein war auch ein erfolgreicher Wissenschaftsmanager: Er verstand es, die Weltsystemanalyse in der US-amerikanischen und globalen Soziologie als eigene Forschungsdisziplin zu verankern. Neben der Diskussion und dem Austausch mit den übrigen Protagonisten der ,gang of four" wurde diese stark durch die Aktivitäten der Soziologie-Fakultät an der Binghampton University (SUNY) geprägt. Dort lehrte und forschte Wallerstein als ,distinguished professor“ und Direktor des Fernand Braudel Center von 1976 bis zu seiner Pensionierung 1999 und seinem anschließenden Wechsel an die Yale University. In diesem Kontext wurden, unterschiedlichen Zeitzeugen zufolge, nächtelang aufreibende Debatten geführt, deren Inhalt heute zuweilen etwas abwegig erscheinen mag: So ging es etwa um die unterschiedlichen historischen Formen, die die Inkorporation des nicht-kapitalistischen Außens annehmen konnte. Aber selbst wenn bei solchen Diskussionen eine gewisse Wagenburgmentalität geherrscht haben mochte, galt Binghampton doch als ein äußerst produktiver Ort, an dem auch waghalsige Ideen realisiert werden konnten. Ein bekanntes Beispiel ist die Datenbank der World Labour Group zu weltweiten Arbeitskonflikten seit dem Jahr 1870, die ohne größere Drittmittelförderung durch den Kreis um Beverly Silver (2003) aufgebaut wurde.

Eine Vielzahl von Wissenschaftler*innen - gerade aus dem globalen Süden arbeitet heute aus einer Weltsystemperspektive $\mathrm{zu}$ sehr unterschiedlichen Themen wie Umweltproblemen, Global Cities oder globalen Ungleichheiten (Babones und Chase-Dunn 2012). Deren rege intellektuelle Produktion füllt zwei wissenschaftliche Zeitschriften („Review“ und ,Journal of World-Systems Research“). Mit der Sektion „Political Economy of the World-System“ wurde die Weltsystemanalyse sogar in der American Sociological Association institutionalisiert. Wallerstein nahm stets an den Sektionssitzungen teil und schuf sich somit sein eigenes wissenschaftliches 
Weltsystem aus Zeitschriften, Konferenzen und Institutionen - mit sich selbst als intellektuellem Vordenker und charismatischem Redner als Zentrum.

In der weltweiten Rezeption des Ansatzes wurde dieser allerdings immer wieder als strukturfunktionalistisch kritisiert: Wallerstein räume Akteuren nur wenig Spielräume innerhalb der Strukturen des Weltsystems ein und führe politische und kulturelle Entwicklungen vorschnell auf ökonomische Faktoren zurück (Skocpol 1977). Ferner waren das den Einfluss von Hegemonialmächten betonende, zyklische Geschichtsbild und das von Robert Brenner (1977) als „,neo-smithianischer Marxismus“ bezeichnete Kapitalismusmodell, das primär auf Austauschverhältnisse im Weltmarkt fokussiere, Gegenstand der Debatte. Ob dieser Kritiken wurden gerade im deutschsprachigen Raum - viele von Wallersteins Verdiensten nur randständig wahrgenommen. Dabei bestand seine vielleicht größte Leistung darin, neue Denkhorizonte eröffnet zu haben. So beeinflusste er auch Diskurse, die heute nicht unbedingt mit seinem Namen verbunden werden: Er spielte etwa eine nicht zu unterschätzende Rolle bei der Entstehung der postkolonialen Studien. Als Vorsitzender der Gulbenkian Commission for the Restructuring of the Social Sciences kritisierte er den historischen Eurozentrismus der Sozialwissenschaften und war Hauptautor des Abschlussberichts Open the Social Sciences, einem der Schlüsseltexte postkolonialen Denkens (Wallerstein et al. 1997). Einen wichtigen Einfluss übte Wallerstein auch auf die Geschichtswissenschaften aus. Selbst wenn sein Werk von Historiker*innen vielfach kritisiert wurde, eröffnete die Weltsystemanalyse globalgeschichtliche Sichtweisen, insbesondere auf die Entwicklung der Weltwirtschaft. Aus der Weltsystemanalyse insgesamt gingen Impulse zur Erforschung von historischen Städtenetzwerken, zu Forschungen zur Frühgeschichte und - beeinflusst von den Arbeiten André Gunder Franks - zur weltwirtschaftlichen Rolle Ostasiens vor der Industrialisierung Europas („California School“) hervor (z. B. Pomeranz 2000). Das Feld der Globalgeschichte wurde somit von Debatten strukturiert, bei denen Wallersteins Werk eine Vorreiterrolle spielte. Wie auch sonst gilt, dass Wallersteins kontrovers diskutierte Thesen ganze Denkräume prägten: Wallerstein war ein Paradigmenmacher.

Neben dem großen Einfluss im wissenschaftlichen Feld verstand Wallerstein sich als öffentlicher Intellektueller, der Konflikte nicht scheute. Seine Parteinahme für progressive und kapitalismuskritische Anliegen kostete ihn zeitweise den Job. Als prominenter Unterstützer der Studierendenproteste 1968 musste er die Columbia University 1971 verlassen und lehrte für fünf Jahre an der McGill University im kanadischen Montreal. Die Weltsystemanalyse war für ihn auch eine Form des politischen Protestes ,gegen die Vernachlässigung von Themen und irreführende Epistemologien“ (Wallerstein 2000, S. XXII). In seinem Spätwerk ging Wallerstein von einer strukturellen Krise des modernen Weltsystems und damit einem Zusammenbruch des globalen Kapitalismus Mitte des 21. Jahrhunderts aus (Wallerstein et al. 2013). Die prophetische Gabe, mit der er, abgeleitet aus den säkularen Trends des Weltsystems, auf das Jahrzehnt genau Zusammenbruchsereignisse vorhersagte, ließ freilich nur spärlichen Raum für historische Kontingenzen. Dem zum Trotz trat Wallerstein immer wieder als politischer Kommentator auf: In insgesamt 500 Kurztexten, die über die Agence Global weltweit vertrieben, in verschiedene Sprachen übersetzt und publiziert wurden, äußerte er sich zu tagespolitischen Themen. Seinen 
ursprünglichen Plan, einen fünften und sechsten Band zu seinem Hauptwerk über das moderne Weltsystem, das nun mit dem Beginn des Ersten Weltkriegs endet, hinzuzufügen, konnte er nicht mehr umsetzen. Immanuel Wallerstein wird eine große Lücke in der soziologischen Diskussion hinterlassen.

Funding Open Access funding provided by Projekt DEAL.

Open Access Dieser Artikel wird unter der Creative Commons Namensnennung 4.0 International Lizenz veröffentlicht, welche die Nutzung, Vervielfältigung, Bearbeitung, Verbreitung und Wiedergabe in jeglichem Medium und Format erlaubt, sofern Sie den/die ursprünglichen Autor(en) und die Quelle ordnungsgemäß nennen, einen Link zur Creative Commons Lizenz beifügen und angeben, ob Änderungen vorgenommen wurden.

Die in diesem Artikel enthaltenen Bilder und sonstiges Drittmaterial unterliegen ebenfalls der genannten Creative Commons Lizenz, sofern sich aus der Abbildungslegende nichts anderes ergibt. Sofern das betreffende Material nicht unter der genannten Creative Commons Lizenz steht und die betreffende Handlung nicht nach gesetzlichen Vorschriften erlaubt ist, ist für die oben aufgeführten Weiterverwendungen des Materials die Einwilligung des jeweiligen Rechteinhabers einzuholen.

Weitere Details zur Lizenz entnehmen Sie bitte der Lizenzinformation auf http://creativecommons.org/ licenses/by/4.0/deed.de.

\section{Literatur}

Amin, S., Arrighi, G., Frank, A. G., \& Wallerstein, I. M. (Hrsg.). (1982). Dynamics of global crisis. New York: Monthly Review Press.

Amin, S., Arrighi, G., Frank, A. G., \& Wallerstein, I. M. (Hrsg.). (1990). Transforming the revolution. Social movements and the world-system. New York: Monthly Review Press.

Arrighi, G. (1994). The long twentieth century. Money, power, and the origins of our times. London: Verso.

Babones, S. J., \& Chase-Dunn, C. (Hrsg.). (2012). Routledge handbook of world-systems analysis. London: Routledge.

Balibar, E., \& Wallerstein, I. M. (1991). Race, nation, class: Ambiguous identities. London: Verso.

Brenner, R. (1977). The origins of capitalist development: A critique of Neo-Smithian Marxism. New Left Review, 18(4), 25-92.

Frank, A. G. (1998). ReOrient: Global economy in the Asian age. Berkeley: University of California Press.

Parsons, T. (1972). Das System moderner Gesellschaften. Weinheim: Juventa.

Pomeranz, K. (2000). The great divergence. China, Europe, and the making of the modern world economy. Princeton: Princeton University Press.

Schmalz, S. (2018). Machtverschiebungen im Weltsystem. Der Aufstieg Chinas und die große Krise. Frankfurt a. M.: Campus.

Schmalz, S., Sommer, B., \& Lütten, J. (2017). Prekarität in der chinesischen Hochwachstumsgesellschaft: Eine Fallstudie zur Situation von Wanderarbeitern im Perlflussdelta. Berliner Journal für Soziologie, 27, 179-207.

Silver, B. (2003). Forces of labor. Workers' movements and globalization since 1870. Cambridge: Cambridge University Press.

Skocpol, T. (1977). Wallerstein's world capitalist system. A theoretical and historical critique. American Journal of Sociology, 82, 1075-1090.

Wallerstein, I. M. (1964). The road to independence: Ghana and the Ivory Coast. Paris: Mouton \& Co.

Wallerstein, I. M. (1979). The capitalist world-economy. Essays. Cambridge: Cambridge University Press. Wallerstein, I. M. (2000). The essential Wallerstein. New York: New Press.

Wallerstein, I. M. (2004) [1974]. Das moderne Weltsystem I. Die Anfänge kapitalistischer Landwirtschaft und die europäische Weltökonomie im 16. Jahrhundert. Wien: Promedia.

Wallerstein, I. M. (2007). World-systems analysis. An introduction. Durham: Duke University Press. 
Wallerstein, I. M. (2011). The modern world-system IV. Centrist liberalism triumphant, 1789-1914. Berkeley: University of California Press.

Wallerstein, I., Collins, R., Mann, M., Derlugian, G., \& Calhoun, C. (2013). Does capitalism have a future? Oxford: Oxford University Press.

Wallerstein, I. M. et al. (1997). Open the social sciences. Report of the Gulbenkian Commission on the restructuring of the social sciences. Stanford: Stanford University Press. 